\title{
Review on Economic Load Dispatch and Associated Artificial Intelligence Algorithms
}

\author{
Swati Jain \\ M. Tech Scholar \\ University Institute of Technology RGPV, \\ Bhopal, M.P, India \\ swatijain260396@gmail.com
}

\author{
Dr Krishna Teeth Chaturvedi \\ Associate Professor \\ University Institute of Technology RGPV \\ Bhopal, M.P, India \\ kteerth@yahoo.co.in
}

\begin{abstract}
In a practical power system, power plants are not equidistant from the distribution center and their fuel cost is different. Under normal operating conditions, the production capacity is even greater than the required total load and losses. Therefore, there are many options for planning the build. In an interconnected network, the objective is to determine the planning of the active and reactive power of each system in order to minimize operating costs. This means that the active and reactive power of the generator can vary within certain limits in order to cover a certain load requirement with minimal fuel costs. This is called the optimal power flow problem. This paper provides an overview of AI-based algorithms, genetic algorithms and their applications with cost-effective charge transport.
\end{abstract}

Keywords: GA, ELD, Neural Network, Power System.

\section{INTRODUCTION}

One of the main problems with commercial electrical systems is the Economic Load Dispatch (ELD). It is defined as a process in which the power of generators is distributed in such a way that the demand for electricity in an electricity grid is met as economically as possible, taking into account any restrictions [1]. The complexity of the ELD problem depends on many factors such as: B. the size of the system, the limits of the system and the characteristics of the generator. Various techniques have been introduced to solve ELD optimization, which can be divided into traditional and stochastic methods. Conventional methods use a deterministic approach such as the LaGrange multiplier, linear programming (LP) and dynamic programming (DP) [2]. These methods have limitations or disadvantages for solving more complex problems. The LaGrange multiplier and LP cannot solve problems with non-linear and unequal properties. The DP method presents a dimensional problem as its memory footprint and execution time increase significantly as the number of generators increases and greater accuracy is required [3]. New techniques have been developed using stochastic approaches to solve optimization problems. Examples are an adaptive Hopfield neural network [4], the simulated annealing method [5] and genetic algorithms (GA).
The problem with ELD is to program the power output for each dedicated production unit in such a way as to minimize operating costs with corresponding operational performance limits, load requirements and various system constraints. The ELD problem is one of the main problems during the operation of a thermal / hydroelectric power plant. It is considered an optimization problem and is defined for minimized total production costs subject to various non-linear and linear constraints to meet electricity demand. The ELD problem is classified in two different ways, the convex ELD problem and the non-convex ELD problem. The convex ELD problem is modeled taking into account the objective function as a minimization of the cost functions of the generator taking into account the linear constraints. The non-convex ELD problem takes into account non-linear constraints / constraints and linear constraints by reducing the cost function. Linear voltages, i. H. Production capacity and power budget make the ELD problem an approximate and simplified problem and the curve is considered piecewise linear. A more precise and precise problem is modeled taking into account non-linear constraints such as prohibited operating zones, valve point effects and ramp speed limits. The problem with ELD is typically multimodal, discontinuous and highly nonlinear. Although the cost curve for heat generating units is generally modeled as a smooth curve, the input / output characteristics are inherently non-linear due to the effects of valve point loading, zones without zones (POZs), ramp speed limits, etc. Turbo generators typically have multiple valves in steam turbines. These valves are opened and closed to maintain an effective balance of forces. However, this effect creates ripples in the cost function. This effect is known as the valve point loading effect. Ignoring the effects of the valve point will result in inaccurate dispensing. Generator sets may also have a specific area where operation is interrupted due to the physical limitations of mechanical components.

The purpose of economic diffusion is to determine the optimal output power of the units involved in powering the load. The sum of the total electricity produced must correspond to the 
load required by the station. In a simplified case, transmission losses are neglected. This simplifies the work of the solution method. In practice, transmission losses must be taken into account. The inclusion of transmission losses makes it more difficult to abandon a low-cost program. Another solution method must be used to arrive at the solution.

\section{INTRODUCTION OF INTELLIGENCE TECHNIQUES}

The rapid growth in the size of the power grid and in the demand for power, as well as the problem of falling operating costs, have become more important as the voltage and thermal safety limits of the power line branches are maintained. A large number of mathematical programs and artificial intelligence techniques have been applied to solve ELD (Economic Load Dispatch). In most general formulations, ELD is a large-scale non-linear, non-convex static optimization problem with continuous and discrete control variables. Mathematical programming approaches the more general formulation. ELD is a large non-linear and non-convex static optimization problem with continuous and discrete control variables.

\section{LITERATURE REVIEW}

Zhi-xinZheng et al. [6] in this study, a hybrid invasive weed optimization (HIWO) algorithm was proposed that hybridizes the invasive weed optimization algorithm and the genetic algorithm (GA) to solve economic shipping problems in electrical systems. In the proposed algorithm, the IWO algorithm is used as the main optimizer to examine the solution space, while the AG crossover and mutation operations are developed to greatly improve the IWO optimization capability. Furthermore, an effective remedial technique is incorporated into the proposed algorithm to correct impractical solutions by addressing various practical limitations of erectile dysfunction problems.

Y. Di et al. [7], the restriction is handled by the edge explorer operator. This method offers an optimal solution for emissions and fuel costs such as BBO and NSGA-II. It has a better overall search ability.

K. Bhattacharjee et al. [8] used the backtracking search algorithm (BSA) and a different strategy for the mutation, using the target individual and the variable in and for a new type of crossing strategy to create new subjects in each generation for a better overall search. Compared to the other method, BSA offers an optimal solution for different generator sets.

A.Y. Abdelaziz et al. [9] had devised a new algorithm called the Flower Pollination Algorithm (FPA) through the process of reproducing flowers from the pollination process. It offers better performance for CEED and ELD problems with a much faster convergence rate.

R M. K. Bavisetti et al. [10] This paper presents an evolutionary computational method (EC) called the Genetic Algorithm (GA) and a metaheuristic algorithm called the Ant Colony Search Algorithm (ACSA) to solve the combined economic transport and emissions (EED) problem with transmission losses. Economic Load Distribution (ELD) and Economic Emission Distribution (EED) were used to achieve optimal fuel costs and optimal emissions from production units. The problem of combined transport of economic emissions is solved by taking into account both economic and emission targets. A real codified GA was created to minimize both shipping costs and emissions in compliance with the constraints linked to equality and inequality.

P.K. Singhal et al. [11] In this paper, an Enhanced Lambda Iteration (ELI) algorithm is developed to solve the problem of cost-effective shipping (ED) of thermal units taking into account the generator limits for a lossless system. With the classic lambda iteration technique, an incorrect selection of the initial lambda value (incremental cost) can lead to slow convergence and therefore divergence. The presented algorithm is able to handle the problem by taking into account the concept of the same incremental cost criterion to determine the value of the initial lambda and also easily handle the equality and inequality constraints. The algorithm is tested on small and large heat production units, which shows the feasibility of the algorithm.

\section{OVER VIEW GENETIC ALGORITHM}

GA starts with a random generation of the initial population, then "Pick", "Cross" and "Mutation" are added until the maximum generation is reached. The important steps of the AG are described below.

\section{A. Selection}

The choice of parents for future generations plays an important role in GA. The goal allows you to choose the strongest, which should be the highest. A group of selection methods is available in the literature [12]: "Universal Stochastic Sampling", "Uniform", "Ranking" and "Tournament" etc. The "Universal Stochastic Sampling" selection is used in this book by "Genetic Algorithm and Direkt" uses the Search Toolbox "in MATLAB. In this selection, parents are created using" Roulette "and" Unified Sample "based on the expectations and the number of parents.

\section{B. Crossover}

Crossover is a major GA operator. It is responsible for structural recombination (exchange of information between mating chromosomes) and the rate of GA convergence and is 
normally used with high probability (0.6-0.9). After the selection process, the one-way crossing will continue. The main purpose of the crossover is to rearrange the information of two different people and create a new one. The Crossover Scattered function is used in this chapter of the Genetic Algorithm and Direct Finder Toolkit in MATLAB. This is a location-independent crossover function that creates crosschildren of the respective population.

\section{Mutation}

Mutation is an underlying operator that causes spontaneous changes in different chromosomes. In artificial genetic systems, the mutation operator protects against irreversible loss. This is an occasional random change of the value in the string position. The mutation is necessary because, although reproduction and crossing are efficient in finding and recombining range terms, they can occasionally lose potentially useful genetic material. This book uses the "Uniform Mutation" Multipoint Mutation feature in the MATLAB Toolkit. The mutated genes are evenly distributed over the area of the gene.

\section{Genetic Algorithm}

GA is a comprehensive stochastic research method that mimics the metaphor of natural biological evolution such as selection, crossing and mutation [13-14]. GA works with string structures, where the string is made up of binary digits that encode the control parameters for a particular problem. All the parameters of the given problem are encoded with bit sequences. The single bit is called "gene" and the content of each gene is called "allele". In general, genetic algorithms consist of three stages: initialization, evaluation and genetic function. The fitness function for the maximization problem is,

\section{$f(x)=F(x)$}

and for the minimization problem is

$$
\mathrm{f}(\mathrm{x})=\frac{1}{1+F(x)}
$$

Where $f(x)$ is fitness function and $F(x)$ is objective function.

In the genetic operation stage, we use genetic operators to generate a new population from the previous population. They are reproduction, crossing and mutation. Reproduction is the operator with which the old chromosome is copied to the carpet pool at the best value. The higher the chromosome match, the higher the number of copies in the next generation chromosome. The different methods of selecting the chromosomes that parents should cross are roulette selection, Boltzmann selection, tournament selection, class selection, steady state selection, etc. The mating tank has a probability proportional to the physical form [15].

The roulette wheel mechanism should make fi / fitavg copies of the i-th chain of the coupling group. The average physical condition is,

$\mathrm{fit}_{\mathrm{avg}}=\sum_{j=1}^{n} \frac{f j}{n}$

The basic operator for creating a new chromosome is the crossover network. This operator is used to exchange information between strings in the array pool to create new strings. The last genetic operator of the algorithm is the mutation. In general evolution, mutation is a random process in which one allele of one gene is replaced by another to create a new genetic structure. Mutation is an important operation because newly created individuals have no new information about heredity and the number of alleles is constantly decreasing.

\section{A. Economic load dispatch}

\section{ECONOMIC LOAD DISPATCH}

Economic shipping can be defined as the process of assigning production steps to production units so that the system load is delivered in full and in the most economical way. For a network system, it is necessary to minimize costs. Economy Freight Shipping is used to define the production level of any asset such that the total cost of production and transportation is minimal for a prescribed load schedule. The goal of economical freight transport is to minimize total production costs.

\section{B. Generator Operating Cost}

Total cost of ownership includes fuel costs, labor costs, supplies and maintenance. Typically, labor, delivery, and maintenance costs are fixed percentages of incoming fuel costs. The performance of fossil fuel power plants is then increased by opening a series of valves on their steam turbine at the entrance. Throttling losses are large when a valve is open and small when fully open.

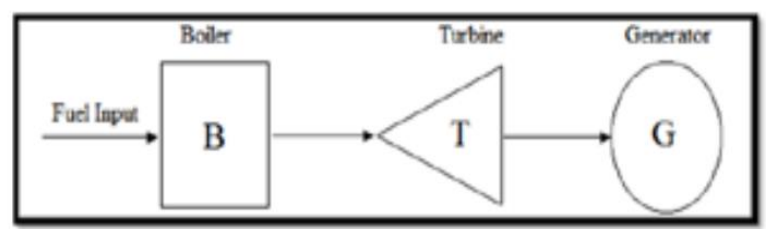

Fig. 1 Simple model of a fossil plant

Figure 1 shows the simple expedition model for fossil plants. Cost is usually estimated from one or more square segments. The operating costs of the plant have the form shown in figure 2. 




Fig. 2 Operating costs of a fossil fired generator

The fuel cost curve can have a series of discontinuities. Discontinuities occur when the power output is increased through the use of additional boilers, steam condensers, or other equipment. They can also arise if the costs represent the operation of an entire system and the costs of connecting generators in parallel therefore lead to discontinuity. In the field of continuity, the additional cost of fuel can be expressed in the number of sections of short line or in linearization by sections. The minimum Pgi is the minimum load limit below which device operation is not economical (or technically impossible), and the maximum Pgi is the maximum output limit.[16].

\section{PRoposed MethodologiEs}

A. Evolutionary Programming (EP), Simulated Annealing (SA), Tabu Search (TS)

Although heuristic methods do not always guarantee the discovery of optimal solutions around the world in a limited time, they often provide a quick and sensible solution. EP can be a rather powerful evolutionary approach; however, for some problems the approach to optimal values is rather slow. SA and TS can be very useful for solving complex reliability optimization problems. However, SA takes time and cannot simply be used to adjust the preheat program control parameters. TS is difficult to define efficient storage structures and problem-dependent strategies.

\section{B. Dynamic Programming (DP)}

If the cost functions are not convex, the same incremental cost method cannot be used. In such circumstances, there is a way to find an optimal broadcast that uses a dynamic programming method. Dynamic programming is an optimization technique that converts a maximization (or minimization) problem with $\mathrm{n}$ decision variables into $\mathrm{n}$ problems with only one decision variable at a time. This is done by defining a sequence of functions with the values V1, V2.....Vn, where an argument $\mathrm{y}$ represents the state of the system. The definition of Vi (y) is the maximum that can be reached when decisions $1,2 \ldots$ I are available and the system state is $\mathrm{y}$. The V1 function is easy to find. For $\mathrm{I}=2$ new system state when this decision is made. Since Vi -1 has already been calculated for the requested states, the above operation returns $\mathrm{Vi}$ for all the requested states. Finally, in the initial state of the system, $\mathrm{Vn}$ is the value of the optimal solution. The optimal values of the decision variables can be restored individually by saving the calculations already performed.

\section{Hopfield Neural Network (HNN)}

Hopfield was introduced in 1982 [17] and 1984 [18]. Hopfield neural networks have been used in many different applications. The important property of the Hopfield neural network is the decrease in energy by a finite quantity as the inputs vary. Hopfield's neural network can then be used for optimization. Tank and Hopfield [19] describe how different optimization problems can be quickly solved by tightly connected networks of a single analog processor which is an implementation of the Hopfield neural network. Park et al. [20] presented convenient freight shipments for piecewise quadratic cost functions using the Hopfield neural network. The results of this method were compared very well with those of the numerical method in a hierarchical approach. King et al. [21] used Hopfield's neural network for economic and ecological transport of electrical systems. However, these applications involved a large number of iterations and often showed oscillations during transients. This suggests that convergence should be improved by an adaptive approach, such as the adaptive learning speed method for a diagonally repeating neural network developed by $\mathrm{Ku}$ and Lee [22].

\section{Genetic Algorithm (GA), Differential Evolution (DE)}

GA ensures that the colony evolves and that solutions are constantly changing. However, sometimes it does not have a strong ability to produce better offspring and causes slow convergence near the global optimum, sometimes it can get trapped in the local optimum. Due to the premature convergence of GA, performance deteriorates and search capability decreases. Price and Storn [23] invented differential evolution (DE). It includes three basic operations: B. Mutation, crossing and selection to obtain an optimal solution. It was found that DE, using its different traversal strategies, offers a better and faster solution and satisfies all the limitations of single and multimodal systems. However, as the system becomes more complex and larger, the DE method cannot better summarize all the unknown variables. In DE, all variables are processed together during the crossover operation. There is no separate vote on the single variable. So in the startup phase the solutions move very quickly to the optimal point, but in a later phase where some fine tuning is needed, DE doesn't work any better.

\section{E. Particle Swarm Optimization (PSO)}


In the mid-1990s, Kennedy and Eberhart invented the PSO [24]. Only a few parameters need to be adjusted in PSO, which makes PSO more attractive. Simple concept, easy implementation, robustness and calculation efficiency are the main advantages of the PSO algorithm. A closer look at how the algorithm works shows that once the algorithm is in the optimal range, it advances slowly because it cannot adjust the speed step size to continue the more precise search. For the multimodal function, particles sometimes do not reach the overall optimal point. Compared to other methods, PSO is inexpensive in terms of storage and speed. The most interesting functions of PSO can be summarized as follows: simple concept, simple implementation, fast calculation and robust search function. Artificial Immune System (AIS) Artificial Immune System (AIS) [25] is another populationbased or network-based soft computer optimization technique that has been successfully implemented in various human health problems: the human system.

\section{F. Bacterial Foraging Algorithm (BFA)}

Inspired by the survival mechanism of bacteria eg. B. E. coli, an optimization algorithm called Bacterial Foraging Algorithm (BFA) has been developed [26]. Chemotaxis, reproduction and dispersion are the three processes by which the full research capability of this algorithm was achieved. These properties have helped BFA to be successfully applied to various types of optimization problems in electrical systems. However, adhering to the restrictions poses few problems with BFA.

\section{G. Quantum-inspired Evolutionary Algorithm (QEAs)}

The quantum-inspired evolutionary algorithms (QEA) [27] then proposed are based on the concepts and principles of the quantum computer, with which it is easier to find the right balance between exploration and exploitation than classical EAs. Meanwhile, QAs can explore the research space with fewer people and use holistic solutions in a short time. In QEA and PSO research, optimization of quantum-inspired particle showers (QPSOs) is suggested. Two main definitions used in QEAs are introduced: quantum bits and quantum rotation gates. The quantum bit is used as a probabilistic representation of particles, which are defined as the smallest unit of information. A quantum bit sequence consists of a single quantum bit. Quantum Rotation Gate is also defined as an implementation to bring individuals to better solutions and ultimately to find a global optimum.

\section{H. Snake Algorithm}

The snake algorithm has been shown to overcome the drawbacks of traditional snake / contour algorithms to follow the most efficient and effective contour of multiple objects. The experimental results of the tests carried out have shown that the proposed method is robust, efficient and precise in order to find solutions at the boundaries of multiple objects.

\section{CONSTRAINED ECONOMIC DISPATCH FORMULATION}

To solve the standard economy shipping problem, consider using a power system with $\mathrm{N}$ units loaded at a time to meet a full load demand, including all transmission losses. Let be the function of the cost of fuel into and out of each unit represented by a function. The units must be loaded in such a way that the total fuel costs for the number $\mathrm{N}$ of production units are minimized depending on the power balance and the upper and lower operating limits of the unit.:

$\min \sum_{i=1}^{N} F_{i}\left(P_{i}\right)$

subject to:

$\sum_{i=1}^{N} P_{i}-\left(P_{D}+P_{L}\right)=0$

$P_{i}^{\min } \leq P_{i} \leq P_{i}^{\max } \quad i=1,2, \ldots, N$

For units with prohibited operating zones, there are additional constraints on the unit operating range:

$P_{i}^{\min } \leq P_{i} \leq P_{i, 1}^{L}$ or

$P_{i, k}^{U} \leq P_{i} \leq P_{i, k}^{L} \quad k=2, \ldots, n_{i}$, or

$P_{i, n_{i}}^{U} \leq P_{i} \leq P_{i}^{\max }$

where:

$i=$ unit index

$P_{i}^{\text {min }}=$ unit minimum generation limit

$P_{i}^{\max }=$ unit maximum generation limit

$n_{i}=$ number of prohibited zones for unit $i$

$k=$ index of prohibited zones of a unit

$P_{i, k}{ }^{L, U}=$ lower/upper bounds of the $k$ th prohibited zones of unit $i$

\section{GENETIC ALGORITHM SOLUTION}

A genetic algorithm [28] represents a class of evolutionary computational techniques based on natural selection and biological evolution. These methods have proved useful in research spaces that are not well understood or that are too large to be researched effectively using standard methods. The 
GA paradigm works with the solution population versus traditional search techniques that work with a single solution. The most powerful feature of GA is that while they don't require any prior information or space constraints such as smoothness or convexity of the feature to be optimized, they work very well on most problems.

\section{A. Real Coded GA}

In this GA, the output of each generator unit is represented by a floating point number instead of a binary encoding, which provides absolute precision, thus eliminating the dependence of precision on string length (number of bits). The outputs of all generators are combined in a chain of solutions called a chromosome. A population of chromosomes is initially generated randomly. Population size is an important parameter of GA and its selection must be made carefully for each problem. Each chromosome in the population represents a possible solution to the problem, and a fitness score derived from the objective function of the problem is then evaluated for each solution chain in the population. Ropes with better solutions receive higher fitness values to ensure their survival into future generations.

\section{B. Parameters of $G A$}

GA seeks better solutions by allowing fitter individuals to take control of the population through a combined stochastic process of selection and recombination. The three main operators influencing GA performance are selection, crossing and mutation. Their interaction is very complex and slight variations in their implementations result in a multitude of models. The different models depend on many factors such as the method and mechanism of selection, the method of parental substitution, the method of crossing and mutation, the implementation in series or in parallel and the type of problem to be solved. The BAC model to be used is selected after a careful analysis of the problem to be solved.

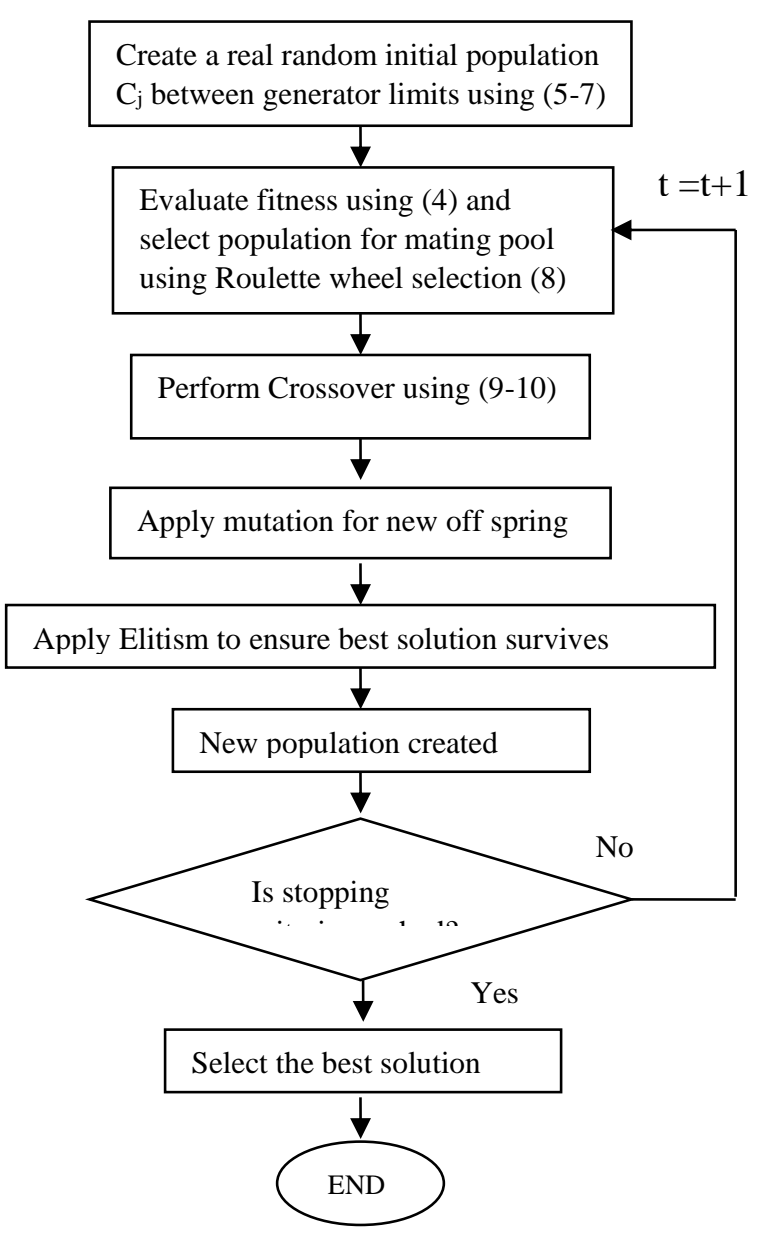

Fig. 1. Flowchart of real-coded genetic algorithm.

\section{Fitness Function}

For the implementation of ELD from RGA, it is necessary that a fitness function is defined so that the operating costs given by (1) are minimized while the constraints given by (2) and (3) are satisfied. One way to do this is the popular penalty function method. In this procedure, the penalty function $t$ consists of quadratic or absolute violations incorporated into the fitness function and set to decrease the value of the chord based on the extent of the injury. High values for penalty parameters alter the penalty function, while very small values do not allow constraint violations to effectively contribute to the punishment of a string. The penalty parameters are then chosen in such a way that an unattainable solution has a lower match than the weakest achievable string of characters. Since two impracticable chains are not treated equally, the chain furthest from the feasibility limit is penalized more severely. Therefore, a constrained optimization problem is converted to an unconstrained optimization problem,

The problem objective function is 


$$
\min \sum_{\mathrm{i}=1}^{\mathrm{N}} \mathrm{F}_{\mathrm{i}}\left(\mathrm{P}_{\mathrm{i}}\right)+\alpha\left[\sum_{\mathrm{i}=1}^{\mathrm{N}} \mathrm{P}_{\mathrm{i}}-\left(\mathrm{P}_{\mathrm{D}}+\mathrm{P}_{\mathrm{L}}\right)\right]^{2}
$$

These are the penalty function for non-compliance with the load requirement and the penalty function for a unit load that falls within a prohibited operating zone. If no unit output in a solution falls within the forbidden zone, the second term of (4) becomes zero.

The fitness function is achieved by converting minimization into maximization.

\section{RGA for generators with prohibited zones}

The goal of an economical shipment is to minimize fuel costs by meeting the unit and system constraints defined in (2) and (3). The system constraint to be observed is to adapt the load demand and the reserve requirement to the production of electricity. The minimum and maximum load limits of the unit are reached through a transformation process that limits the solutions to these values [29]. So the only other restrictions to consider are the prohibited areas of operation of the unit. An approach based on the penalty function $[12,6]$ is used to overcome these limitations. Another popular approach is to set the solutions to the next upper or lower limit of the forbidden zone that is violated.

The following steps describe how GA handles the large solution space and achieves solutions very close to the overall minimum.

\section{$\underline{\text { Step I: }}$ Generation of initial population}

A population of $\mathrm{M}$ chromosomes is randomly generated in which each generator output $\mathrm{P}_{\mathrm{i}}$ is constrained to lie within $P_{i}^{\min }$ and $P_{i}^{\max }$ [29]. The bounded constraints are handled as unbounded constraints by transformation of variables.

$$
\begin{aligned}
& \text { Let } \quad \mathrm{A}=\left(P_{i}^{\min }+P_{i}^{\max }\right) / 2 \\
& \mathrm{~B}=\left(P_{i}^{\max }-P_{i}^{\min }\right) / 2
\end{aligned}
$$

Then the transformed variable $\mathrm{p}^{\mathrm{t}}$ is found $P^{t}=(A+B \operatorname{Cos}(r \pi))$

The $\mathrm{j}^{\text {th }}$ chromosome in the initial population can be represented as

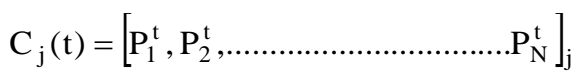

where $\mathrm{j}$ represents the population size $\mathrm{M}$.

$\underline{\text { Step II: Evaluation of fitness function }}$
ELD's goal is to minimize operating costs by meeting demand and other constraints. The RGA works across the entire population to maximize fitness, so the fitness function is evaluated as the inverse of the function (4). Therefore, for each chromosome of the generated population, the fitness function is annotated using the inverse of the objective function given by (4)

\section{Step III: Evolution of population to next generation}

The initial population evolves using the three basic genetic operators: selection, crossing and mutation. To this end, the chromosomes of the parental population are copied into the mating pool with a probability proportional to their suitability to form the offspring population using the roulette wheel. [28].

The probability of selection of the $i^{\text {th }}$ string is given by

$$
\rho_{i}=\frac{F_{i}}{\sum_{j=1}^{M} F_{j}}
$$

Where $\mathrm{M}$ is the population size.

Step IV: Creation of offspring through crossover operator

So far, the channels have only received reruns with no new additions. The crossing operation is used to create new people with better physical shape. In binary GA, some of the parents are swapped with each other in random positions. The crossing probability controls the crossing frequency in a generation. This is achieved by swapping two high-level chains in randomly chosen locations. For a continuous real encoded GA, if two chromosomes $\mathrm{C} 1(\mathrm{t})$ and $\mathrm{C} 2(\mathrm{t})$ are randomly chosen from the population for crossing, the two secondary feathers $\mathrm{C} 3(\mathrm{t})$ and $\mathrm{C} 4(\mathrm{t})$ are indicated as,

$$
\begin{aligned}
& C_{3}(t+1)=r C_{1}(t)+(1-r) C_{2}(t) \\
& C_{4}(t+1)=r C_{2}(t)+(1-r) C_{1}(t)
\end{aligned}
$$

Where $\mathrm{t}$ represents generation and $\mathrm{r}$ is a random positive number; $r \in[0,1]$.

\section{$\underline{\text { Step V: Mutation operation }}$}

The two operators above, crossing and reproduction, have efficiently recombined existing chromosomes, but no new genetic information is added to the pool. The mutation operator changes the alleles (individual members of the chromosome, i.e. the outputs of the generators) randomly. If the $\mathrm{i}$-th variable of the $\mathrm{m}$-th chromosome is randomly chosen for the mutation of a population of $\mathrm{M}$, it is the newly created chromosome, 


$$
C_{m}^{\prime}(t+1)=\left[P_{1}^{t}, P_{2}^{\prime} \ldots \ldots \ldots \ldots \ldots P_{m}^{t-}, \ldots \ldots \ldots P_{N}^{t}\right]
$$

such that $P_{m}^{t-}$ lies between $P_{m}^{M a x}$ and $P_{m}^{\text {Min }}$

A simple RGA treats the mutation as a secondary operator. The mutation operator introduces new genetic structures into the population by randomly modifying some of its constituent elements. Help the search algorithm escape local minima.

The probability of crossing and mutating in a race is appropriately selected. These settings have a noticeable effect on RGA convergence.

\section{Step VI: Elitism for preserving the best solutions}

As the population moves through the research space under the guidance of genetic operators, it is likely that the best chains of solutions, i. H. Strings with high fitness values are lost. Eliteism ensures that some of the best channels from previous generations are copied unchanged into the new generation.

\section{$\underline{\text { Step VI: Stopping criteria }}$}

The RGA algorithm is applied to the randomly generated population to maximize the fitness function. The stopping criterion used is after a predetermined number of generations, or when the ratio of minimum to maximum ability in a population approaches one, indicating saturation.

\section{CONCLUSION}

This paper describes artificial intelligence algorithms and their associations with the transport of economic loads. Due to its attractive features, GA has become very popular for use in various power system applications, including ELD. Many articles have been reviewed on the use of GA to solve the problem of ELDs. ELD problems of varying complexity have been studied in the literature using GA with satisfactory results. Economic Load Balancing (ELD) is a process of finding an optimal production plan for generators available in an interconnected network to meet system requirements at the lowest possible cost and at the same time to meet various operating system restrictions. More specifically, the software's calculation method has received additional attention and has been used in a number of useful and successful applications. Here we try to find out the minimum cost using various algorithm techniques using data from some production units.

\section{REFERENCES}

[1] Chowdhury, B.H. and Rahman, S. A review of recent advances in economic dispatch. IEEE transaction on power system. Nov. 1990. Vol. 5. No. 4. pp. 1248-1259

[2] Deschamps, D .. Op timization in power system planning. I n: ElAbiad, AH. Ed. Power system analysis and planning. London: Hemishpere Publishing Corporation. 1981. Pp.201-208
Bakirtzis, A. etal. Genetic algorithm solution to the economic dispatch problem. IEE proceedings- genera tion, transmission and distributio n. Jul 1994. Volume: 141. Issue: 4. pp.: 377-382

Lee, K.Y. etal. Adaptive hopfield neural network for economic dispatch. IEEE transactions on power systems. Vol. 1 8, NO. 2. Feb. 2003 pp. $519-529$.

[5] Simopoulos, D., and Contaxis, G. 2004. Unit commitment with ramp rate constraint $\mathrm{u}$ sing stimulated annealin $\mathrm{g}$ algorithm. IEEE Melecon. May 12-15. Dubrovnik, Croatia. Pp. 845-849.

[6] Zhi-xinZheng, Jun-qing Li, Hong-yan Sang. A hybrid invasive weed optimization algorithm for the economic load dispatch problem in power systems[J]. Mathematical Biosciences and Engineering, 2019, 16(4): 2775-2794. doi: 10.3934/mbe.2019138.

[7] Di Y, Fe M, Wang L, Wu W. Multi-objective optimization for economic emission dispatch using an improved multi-objective binary differential evolution algorithm. Energy Procedia; 2014, 61:p.2016-2021.

[8] Bhattacharjee K, Bhattacharya A, Dey SH. Backtracking search optimization based economic environmental power dispatch problems. Electrical Power and Energy Systems; 2015, 73: 830-842.

[9] Abdelaziz AY, Ali ES, Elazim SM. Combined economic and emission dispatch solution using Flower Pollination Algorithm. Electrical Power and Energy Systems; 2016, 80: 264-274.

[10] R M. K. Bavisetti and T. KranthiKiran, "Optimization Of Combined Economic and Emission Dispatch Problem - A Comparative Study for 30 Bus Systems." IOSR Journal of Electrical and Electronics Engineering (IOSR-JEEE) 2012; Vol 2, pp. 37-43.

[11] P.K. Singhal, R. Naresh, V. Sharma and N. Goutham Kumar, "Enhanced lambda iteration algorithm for the solution of large scale economic dispatch problem", Recent Advances and Innovations in Engineering (ICRAIE), 2014, vol., no., pp. 1, 6, 9-11 May 2014. doi: https://doi.org/10.1109/ICRAIE.2014.6909294.

[12] X.P. Wang, L.P. Cao, Genetic Algorithms - Theory, Application and Software Realization, Xi'an Jiaotong University, Xi'an, China, 1998. http:// en.wikipedia.org/wiki/Genetic_algorithm

[14] Mitchell M., “An Introduction to Genetic Algorithm”, MIT Press, 1998.

[15] Singh S. P., Bhullar S., "Hybrid Approach to Economic Load Dispatch", National Conference on Artificial Intelligence and Agents: Theory \& Applications, IIT (BHU) Varanasi, Dec. 2011.

[16] Arunpreet Kaur, Harinder Pal Singh, Abhishek Bhardwaj, “Analysis of Economic Load Dispatch Using Genetic Algorithm”,Interational Journal Of Application or Innovation in Engineering \& Management, Volume 3,Issue 3, March 2014.

[17] Sulaiman M.H., Mustafa M.W., Zakaria Z.N, Aliman O. and Rahim A. S. R., "Firefly Algorithm Technique for Solving Economic Dispatch Problem", IEEE International Power Engineering and optimization conference, Malaysia, 6-7, , pp. 90-95, June, 2012.

[18] Amoli N.A., Jadid S., Shayanfar H. A., Barzipour F., "Solving Economic Dispatch Problem With Cubic Fuel Cost Function by Firefly Algorithm", ICTPE conference on Technical Physical Problems of Power Engineering, vol. 1, pp 1-5, 2012.

[19] YounesMimoun , "Environmental/Economic power Dispatch Problem/ Renewable Energy Using Firefly Algorithm”, International Conference on Environmental, Energy, Ecosystem and Development, pp 170-176, 2013.

[20] Swarnkar K.K., AS.P, "Economic load Dispatch Problem with Reduce Power losses using Firefly Algorithm", Journal of Advanced computer science and technology, vol. 1, pp 42-56, 2012.

[21] Niknam T., Azizipanah A., Roosta R., "Reserve Constrained Dynamic Economic Dispatch: A New Fast Self-Adaptive Modified Firefly Algorithm", Systems Journal, vol.6, no.4, pp. 635- 646, December 2012.

[22] Apostolopoulos T. and Valchos A., "Application of Firefly Algorithm for Solving the Economic Emission Load Dispatch Problem", 
International Journal of Combitronics, volume 2011. 23 pages, doi: $10.1155 / 2011 / 523806$.

[23] Reddy S. and Reddy M. D., "Economic Load Dispatch Using Firefly Algorithm", International Journal of Engineering Research and Application, vol. 2, issue 4, pp. 2325 2330, July 2012.

[24] Abedinia O., Amjady N., Naderi M.S., "Multi-objective Environmental/Economic Dispatch using firefly technique," 11 th International Conference on Environment and Electrical Engineering (EEEIC), 18-25 May 2012, pp. 461-466, 2012.

[25] Sulaiman M. H., Daniyal H., Mustafa M. W., “ Modified Firefly Algorithm In Solving Economic Dispatch Problem With Practical Constraints", IEEE International Conference on Power and Energy, PECon, Kota Kinabalu, Malaysia, pp. 157- 161, 2012.

[26] Dekhici L., Belkadi K., Lamosi B. P., “ Firefly Algorithm for Economic Power Dispatching With Pollutants Emission", ImformaticaEconomica, vol. 16, issue 2, pp 45 57, 2012.

[27] Rao C.V. G.K. and Yesuratnam G., "Big Bang And Big Crunch And Firefly Algorithm Application And Comparison To Optimal Power Flow With Continuous And Discrete Control Variables", International Journal On Electrical Engineering And Informatics, vol. 4, issue 4, pp. 575-583, December 2012.

[28] D.E.Goldberg, "Genetic algorithms in search, optimization and machine learning”, (Book ), Addison-Wesley, 1989.

[29] Randy L.Haupt and Sue Ellen Haupt, "Practical genetic algorithms", (Book ), John Wiley and sons, second edition, 2004 